\title{
Energy Efficiency-Aware Joint Resource Allocation and Power Allocation in Multi-User Beamforming
}

\author{
Xuanli Wu ${ }^{\circledR}$, Member, IEEE, Zheming Ma, Xu Chen ${ }^{\circledR}$, Fabrice Labeau ${ }^{\circledR}$, Senior Member, IEEE, \\ and Shuai Han (1), Senior Member, IEEE
}

\begin{abstract}
As a conventional metric, spectrum efficiency has been investigated widely in communication systems due to the limited bandwidth resources and increasing subscriber density. Beamforming technology is used in TD-LTE-A and $5 \mathrm{G}$ downlink to improve the spectrum efficiency and system capacity, and a proper resource allocation algorithm can also be used to improve the spectrum efficiency on the premise of satisfying user equipment (UE) requirements. In recent years, energy efficiency has attracted more and more attention as the power consumption on the communication systems increases rapidly, which lays a heavy burden on the environment. In this paper, we propose a joint resource allocation and power allocation algorithm in multi-user beamforming mode, which aims at maximizing the energy efficiency, and also takes UE requirements and spectrum efficiency into consideration. The proposed algorithm first calculates the user priorities and gives a UE grouping method to allocate resource blocks (RBs) to different UEs under the assumption that the power is equally allocated among UEs on the same RB. After that, on each RB, it calculates the power allocation among different UEs to realize the improvement of energy efficiency. In addition, the satisfaction of UE requirements is added in the algorithm as a constraint. Simulation results demonstrate that the proposed algorithm shows an excellent performance on system capacity, i.e., spectrum efficiency, and also provides higher energy efficiency than existing algorithms. Moreover, the UE requirements can be better satisfied and system fairness can also be improved.
\end{abstract}

Index Terms-Resource allocation, power allocation, MUMIMO, energy efficiency, UE grouping, beamforming.

\section{INTRODUCTION}

A S A KEY technology in Long Term Evolution Advanced (LTE-A) system, multiple input multiple output (MIMO) technology shows excellent performance on peak data rate, spectrum efficiency and latency [1], and will continue playing an important role in $5 \mathrm{G}$ networks [2]. With the rapid development of

Manuscript received September 8, 2018; revised January 18, 2019; accepted March 4, 2019. Date of publication March 15, 2019; date of current version May 28, 2019. This work was supported in part by the National Basis Research Program of China 973 Program under Grant 2013CB329003 and in part by the National Natural Science Foundation of China under Grants 61671179 and 61301100 . The review of this paper was coordinated by Dr. Z. Fadlullah. (Corresponding author: Shuai Han.)

$\mathrm{X}$. Wu, X. Chen, and S. Han are with the Communication Research Center, Harbin Institute of Technology, Harbin 150006, China (e-mail: xlwu2002@hit.edu.cn; xchen1997@163.com; hanshuai@hit.edu.cn).

Z. Ma was with the Communication Research Center, Harbin Institute of Technology, Harbin 150006, China. He is now with Ericsson (China) Communications Co., Ltd, Beijing 100004, China (e-mail: mr_mzm_4939@163.com).

F. Labeau is with the Department of Electrical and Computer Engineering, McGill University, Montreal QC H3A 0C8, Canada (e-mail: fabrice.labeau@mcgill.ca).

Digital Object Identifier 10.1109/TVT.2019.2905251 wireless communications, improving spectrum efficiency and energy efficiency have been challenging issues in future networks. Because of the scarce bandwidth resources [3], there have been many literatures focusing on the spectrum efficiency [4]. On the other hand, energy efficiency is attracting more and more attention recently due to the reason that the power consumption in telecommunications is increasing rapidly, which lays a heavy burden on environment. Ref. [5] and [6] discussed the relationship between energy efficiency and spectrum efficiency, and demonstrated that MIMO technology can help to increase the spectrum efficiency and energy efficiency simultaneously. Beamforming algorithms for MIMO technology, e.g., zero-forcing (ZF) and block-diagonalization (BD), which can significantly reduce the interference and improve the signalto-interference-plus-noise, are widely used in recent research work [7], [8]. Especially, the multi-user MIMO (MU-MIMO) can eliminate the interference via its high spatial degree of freedom, and thus, our paper will focus on the MU-MIMO scenario.

Besides beamforming algorithm, resource allocation and power allocation algorithms will also affect system performance. Metrics of these algorithms include spectrum efficiency, fairness, satisfaction to UE Quality of Service (QoS), energy efficiency, and etc. How to design a resource allocation algorithm which can improve spectrum efficiency and energy efficiency comprehensively needs to be considered, since they are not always consist with each other [9]. Resource allocation can effectively reduce co-channel interference and improve spectrum efficiency, which is investigated by many researchers. Ref. [10] investigated the resource allocation and power allocation algorithms with the objective to maximize system spectrum efficiency. Ref. [11] studied a resource allocation algorithm with the consideration of co-channel interference mitigation and fairness. Ref. [12] presented an algorithm to find the optimal transmit power to maximize the spectrum efficiency. However, these algorithms do not pay enough attention to UE requirements which is critical issue for communication networks [13]. Moreover, the power consumption of such algorithms is extremely high, which causes low energy efficiency against the concept of green communications. Ref. [14] minimized the energy consumption while satisfying the quality of service $(\mathrm{QoS})$ requirement in the system to realize green communications. Nevertheless, such objective function may not realize the optimal energy efficiency. Algorithms in [15] and [16] introduced the energy efficiency optimized power allocation algorithms, which improve the energy efficiency without the consideration of spectrum efficiency. 
However, high realization complexity and high signaling overhead is required for such proposed algorithms. Ref. [17] investigated the classical model of energy efficiency optimized power allocation and gives the optimal solution with low complexity, however only the optimization of energy efficiency is considered. The algorithm in [18] considers the energy efficiency and spectrum efficiency with low complexity, however the authors do not take the fairness and data rate requirement into consideration. Ref. [19] investigated the problem of QoE and energy aware small cell base station (SBS) management consisting of power selection, load management and resource allocation. Ref. [20] investigated an energy-efficient power allocation and resource allocation algorithm considering QoS requirements for IoT systems in $5 \mathrm{G}$ era. However, despite meeting the data rate requirement contained by QoS, the algorithms in [19] and [20] did not consider the fairness, which is another important performance metric of communication system.

To the best of our knowledge, there is no existing work about resource allocation and power allocation considering the performance metrics including spectrum efficiency, energy efficiency, data rate requirement and fairness comprehensively. To solve this problem, we propose an energy efficiency aware joint resource allocation and power allocation algorithm considering such performances based on ZF beamforming technology in multi-user MIMO scenario. The proposed algorithm can obtain high energy efficiency on the premise that the UEs' different data rate requirements can be satisfied. In the first phase, the proposed algorithm allocates the power equally to all the resource blocks (RBs) as well as the UEs on the same RB, and selects the proper UE group on each RB, which considers both the channel conditions and the co-channel interference. Then, the power of different UEs in the same group will be reallocated to further improve the energy efficiency of the system. During the whole process, the fairness is also considered. Simulation results show that compared with other algorithms and our former work, the proposed algorithm can realize high energy efficiency and ensure an acceptable spectrum efficiency in terms of the satisfaction of different data rate requirement. Moreover, the highest fairness can also be obtained by our proposed algorithm.

The contribution of this paper can be summarized as follows:

- We investigate the problem of UE grouping and resource allocation in multi-user MIMO scenario and propose a UE grouping method with the consideration of co-channel interference and channel conditions of different UEs to improve the energy efficiency with the constraint of satisfying the requirement of different UEs. Comparing with the conventional resource allocation methods, our proposed algorithm can reduce the interference within one RB and satisfy the requirement of different UEs at the same time, and the energy efficiency can also be improved.

- Then, we focus on the power reallocation for each UE group. With the ZF beamforming, an adjusted water-filling based power allocation algorithm is applied in each UE group after the completion of spectrum resource allocation to further improve the energy efficiency of the whole system. Hence, after reducing co-channel interference, transmitted power can be used effectively and better energy efficiency can be achieved.
- Combining the above two algorithms, we can realize the optimization of energy efficiency considering the spectrum efficiency and UEs' different data rate requirement. Meanwhile, we also consider the fairness during the resource allocation and power allocation process so that the fairness can be improved by the proposed joint optimization algorithm.

The remainder of this paper is outlined as follows. Section II introduces the system model and problem formulation. Section III presents the joint resource allocation and power allocation algorithm. Performance of the proposed algorithm is evaluated by simulations in Section IV. And finally, Section V concludes the paper.

\section{System Model AND PROBlem Formulation}

In this paper, we consider the downlink of a LTE-A system with $N_{t}$ transmit antennas on the eNodeB, and $K$ active UEs in the system, each with a single receive antenna. The system has $N$ RBs, and each RB consists of 12 subcarriers (total bandwidth of $180 \mathrm{kHz})$ in frequency domain and one time slot $(0.5 \mathrm{~ms})$ in time domain. The RBs will be allocated to different UEs based on a comprehensive consideration, e.g., the channel conditions and the QoS requirement of different UEs.

In the receiver end of UE $k$, the received signal $\mathbf{y}_{k, n}$ can be expressed as:

$$
\begin{aligned}
\mathbf{y}_{k, n}= & p_{k, n} \mathbf{H}_{k, n} \mathbf{W}_{k, n} \mathbf{X}_{k} \\
& +\sum_{j=1, j \neq k}^{K} \rho_{j, n} p_{j, n} \mathbf{H}_{k, n} \mathbf{W}_{j, n} \mathbf{X}_{j}+\mathbf{n}_{k, n},
\end{aligned}
$$

where, $\mathbf{W}_{k, n} \in \mathbb{C}^{N_{t} \times 1}$ is the normalized beamforming matrix of UE $k$ on RB $n, \mathbf{X}_{k}$ is the normalized transmitted signal of UE $k$, and $\mathbf{n}_{k, n}$ is the white Gaussian noise with zero mean and variance $\sigma^{2} . \mathbf{H}_{k, n}$ represents the transmission channel matrix from the eNodeB to UE $k$ on RB $n$. The parameter $p_{k, n}$ is the transmit power allocated to UE $k$ on RB $n$, and $\rho_{k, n}$ is an allocation indicator, such that $\rho_{k, n}=1$ when $\mathrm{RB} n$ is allocated to UE $k$ and $\rho_{k, n}=0$ otherwise.

Then, the energy efficiency of UE $k$ on RB $n \eta_{k, n}^{e e}$ can be defined by the following expression [17]:

$$
\eta_{k, n}^{e e}=\frac{C_{k, n}}{P_{k, n}^{t o t}}=\frac{B \log _{2}\left(1+\gamma_{k, n}\right)}{p_{k, n}+p_{n}^{(C)}},
$$

where, $C_{k, n}$ represents the channel capacity that UE $k$ can obtain on RB $n$, and $P_{k, n}^{t o t}$ is the total power consumption of UE $k$ on RB $n$, which includes $p_{k, n}$ and $p_{n}^{(C)}$, where $P_{n}^{(C)}$ is circuit power consumed on each RB $n . C_{k, n}$ is calculated by the Shannon equation, where $B$ is the bandwidth of each $\mathrm{RB}$, and $\gamma_{k, n}$ is the SINR of UE $k$ on RB $n$, which can be calculated by:

$$
\gamma_{k, n}=\frac{p_{k, n}\left\|\mathbf{H}_{k, n} \mathbf{W}_{k, n}\right\|_{F}^{2}}{\sum_{j=1, j \neq k}^{K} \rho_{j, n} p_{j, n}\left\|\mathbf{H}_{k, n} \mathbf{W}_{j, n}\right\|_{F}^{2}+\sigma^{2}},
$$

where, $\sum_{j=1, j \neq k}^{K} \rho_{j, n} p_{j, n}\left\|\mathbf{H}_{k, n} \mathbf{W}_{j, n}\right\|_{F}^{2}$ represents the interference from other UEs, and $\|\cdot\|_{F}$ represents the Frobenius norm. 
Then, the energy efficiency of the whole system can be calculated by the following formulation:

$$
\eta^{e e}=\frac{\sum_{k=1}^{K} \sum_{n=1}^{N} \rho_{k, n} B \log _{2}\left(1+\gamma_{k, n}\right)}{\sum_{k=1}^{K} \sum_{n=1}^{N} \rho_{k, n} p_{k, n}+P^{(C)}},
$$

where, $P^{(C)}$ is the total circuit power consumption which is the sum of circuit power consumed on all RBs, and can be calculated by $P^{(C)}=\sum_{1}^{n} P_{n}^{(C)}$.

Besides the energy efficiency, we also need to define the spectrum efficiency of the system.

The spectrum efficiency of UE $k$ on RB $n \eta_{k, n}^{s e}$ can be obtained as:

$$
\eta_{k, n}^{s e}=\frac{C_{k, n}}{B}=\log _{2}\left(1+\gamma_{k, n}\right) .
$$

From [21], we can know that the relationship between energy efficiency and spectrum efficiency is non-monotonic. With the spectrum efficiency going up, the value of energy efficiency will firstly increase and then displays a decreasing trend when $P^{(C)}$ is a fixed value. Optimization on single metric is not sufficient in a future $5 \mathrm{G}$ system since energy efficiency and spectrum efficiency are not always consistent with each other. Hence, we need to make a trade-off between such two targets. Designing the combination of energy efficiency and spectrum efficiency as the objective function seems feasible, however, such algorithm will make it difficult to obtain the optimal solution due to the complicated form of the objective function. To make the problem solvable with moderate realization complexity, we formulate this problem with the objective function of energy efficiency and take the spectrum efficiency as a constraint. Then, the problem can be formulated as:

$$
\max _{\rho_{k, n}, p_{k, n}} \eta^{e e}
$$

subject to:

$$
\begin{aligned}
& \rho_{k, n} \in\{0,1\}, \forall n \in \mathcal{R}, \forall k \in \mathcal{U}, \\
& \sum_{k=1}^{K} \rho_{k, n} \leq N_{t}, \forall n \in \mathcal{R}, \\
& \sum_{n=1}^{N} C_{k, n} \geq \lambda_{k}, \forall k \in \mathcal{U}, \\
& \sum_{k=1}^{K} \sum_{n=1}^{N} \rho_{k, n} p_{k, n} \leq P_{T},
\end{aligned}
$$

where, $\mathcal{U}=\{1,2, \ldots, K\}$ is the active UE set, $\mathcal{R}=$ $\{1,2, \ldots, N\}$ is the RB set, $\lambda_{k}$ is the target data rate of UE $k$ depending on the QoS requirements, and $P_{T}$ is the maximum transmit power. Constraint (6c) restricts the number of UEs on a single RB, and since we consider the scenario with only one receive antenna in the UE end, the number of data layers is just the same as the co-existed active UE number on the same RB. Constraint (6d) shows the limitation of the minimum data rate to guarantee the requirement of different UEs. The maximum transmit power restriction is given in (6e).

\section{Joint Resource AlLocation AND POWER ALLOCATION ALGORITHM}

In Section III, we focus on the solution to the problem (6) formulated in last Section. The problem is a non-convex problem, which can obtain the optimal solution by exhaustive search algorithm with huge amount of computation. Moreover, the constraint for spectrum efficiency further increases the realization complexity, which cannot be easily solved by conventional algorithms. To simplify the problem, we decompose the problem into two sub-problems, i.e., resource allocation and power allocation.

\section{A. Resource Allocation}

In this Sub-Section, we focus on the solution of $\rho_{k, n}$ to obtain the result of resource allocation.

Different UEs will experience different channel conditions on different RBs. With a fixed transmit power, UEs with high channel quality on a specific RB can show more excellent performance, no matter in terms of energy efficiency or spectrum efficiency. To improve system performance, allocating proper RBs to UEs is necessary. Besides the allocation relationship between UEs and RBs, UE grouping, which determines the co-channel interference among different UEs on one RB, also plays an important role. A reasonable UE grouping result can reduce the co-channel interference due to small spatial correlation. However, in LTE-A system, UEs cannot calculate the co-channel interference before knowing the beamforming matrix, resource allocation results and power allocation results [22], which makes it difficult to determine the UE grouping result.

Similar to our former work [22], we use spatial correlation indicator to represent the co-channel interference between two UEs without acquiring the beamforming matrix and signal detection algorithm. Then, we propose a UE grouping and resource allocation algorithm based on the spatial correlation indicator to solve the above problem. The spatial correlation indicator is defined as follows:

$$
\eta_{l, k, n}=\frac{\left|\mathbf{H}_{l, n}\left(\mathbf{H}_{k, n}\right)^{H}\right|}{\left\|\mathbf{H}_{l, n}\right\|_{F}\left\|\mathbf{H}_{k, n}\right\|_{F}},
$$

where, $\eta_{l, k, n}$ represents the spatial correlation indicator between UE $l$ and UE $k$ on RB $n$. It describes the degree of similarity between channel matrixes of these two UEs, and can be used to evaluate the inter-user interference [22], [23]. Based on this indicator, we can group UEs properly with a comprehensive consideration of interference and UE requirements. The whole algorithm of resource allocation consists of the following phases:

1) All the UEs waiting for RB allocation can be organized as a set $\mathcal{U}_{\mathrm{w}}$. The power on each RB is allocated equally to UEs on this $\mathrm{RB}$ in the resource allocation process.

2) Based on the obtained channel matrix, select UE $k^{*}$ on RB $n^{*}$ by $k^{*}=\arg \max \left(\gamma_{k, n *}^{\prime}\right)$, where $\gamma_{k, n *}^{\prime}$ is the SINR of UE $k$ on $\mathrm{RB} n^{*}$ in last TTI. Then, we put UE $k^{*}$ into the UE set of RB $n^{*} \mathcal{A}_{\mathrm{n} *}$, and set $\rho_{k^{*}, n^{*}}=1$. 
3) Calculate the average spatial correlation between the UEs assigned to $\mathrm{RB} n^{*}$ and the other UEs waiting for resource allocation as follows:

$$
\overline{C_{m, n^{*}}}=\frac{\sum_{l \in \mathcal{A}_{n^{*}}} \eta_{l, m, n^{*}}}{\operatorname{card}\left(\mathcal{A}_{n^{*}}\right)}, \forall m \in \mathcal{U}_{w},
$$

where, $\overline{C_{m, n^{*}}}$ is the average spatial correlation indicator between UE $m$ and the UEs which have already been assigned to $\mathrm{RB} n^{*}$, and card $\left(\mathcal{A}_{\mathrm{n} *}\right)$ calculates the number of elements in set $\mathcal{A}_{\mathrm{n} *}$. Then, find $L$ UEs with the smallest $\overline{C_{m, n^{*}}}$, where $L=\min \left\{\operatorname{card}\left(\mathcal{U}_{\mathrm{w}}\right), N_{t}\right\}$ is used to realize the trade-off between interference and channel condition. In other words, we should not decide the UE grouping and RB allocation scheme monolithically through the best channel condition or the lowest cochannel interference. In the following parts, we will explain how to realize the tradeoff using $L$.

After finding the $L$ UEs, the UE $m^{*}$ with better channel condition and less interference will be selected, i.e., we choose UE $m^{*}$ out of the $L$ UEs which can maximize the sum channel capacity on $\mathrm{RB} n^{*}$ and set $\rho_{m^{*}, n^{*}}=1$. Next, repeat the procedure until the number of UEs on the RB reaches the constraint, as described in (6c). If no UE can be found to improve the sum channel capacity on $\operatorname{RB} n^{*}$, the allocation on $\operatorname{RB} n^{*}$ will be terminated. Hence, UEs with lower interference and better channel quality are grouped on RB $n^{*}$ by this method.

4) After the resource allocation procedure in (1) to (3) and the power allocation on $\mathrm{RB} n^{*}$ in Sub-Section B are finished, we can calculate the channel capacity and spectrum efficiency of all the UEs. If $\Sigma_{n=1}^{N} B \eta_{k, n}^{e e} \geq \lambda_{k}$, i.e., UE $k$ can satisfy (6d), it will be removed from UE set $\mathcal{U}_{\mathrm{w}}$ in this TTI. Otherwise, it will go on to take part in the following allocation in other RBs.

5) Repeat (2) to (4) until all the RBs are allocated properly.

In this resource allocation Sub-Section, we propose a UE grouping method and determine the UE assignments on different RBs considering the co-channel interference and channel conditions comprehensively, which is helpful to improve the energy efficiency performance of UEs. Moreover, the constraint of spectrum efficiency is also considered by selecting the UE which can maximize the sum channel capacity in phase (3).

\section{B. Power Allocation}

The resource allocation results are obtained in the previous Sub-Section based on equal power allocation among UEs on the same RB. In this Sub-Section, we will reallocate the power to UEs on the same RB to increase the energy efficiency. The objective function of the power allocation on $\mathrm{RB} n^{*}$ can be described as:

$$
\max _{\mathbf{P}_{k, n^{*}}} \frac{\sum_{k \in \mathcal{A}_{n^{*}}} B \log _{2}\left(1+\gamma_{k, n^{*}}\right)}{\sum_{k \in \mathcal{A}_{n^{*}}} p_{k, n^{*}}+P_{n^{*}}^{(C)}}
$$

where, $\mathbf{P}_{k, n^{*}}=\left[p_{1, n^{*}}, p_{2, n^{*}}, \ldots, p_{k, n^{*}}\right]$ is the power allocation vector, $P_{n^{*}}^{(C)}$ is the circuit power consumption on $\operatorname{RB} n^{*}$, which is calculated by $P_{n^{*}}^{(C)}=\sum_{k \in \mathcal{A}_{n^{*}}} p_{k, n^{*}}^{(C)}$, and $\gamma_{k, n^{*}}$ is calculated by:

$$
\gamma_{k, n^{*}}=\frac{p_{k, n^{*}}\left\|\mathbf{H}_{k, n^{*}} \mathbf{W}_{k, n^{*}}\right\|_{F}^{2}}{\sum_{j \in \mathcal{A}_{n^{*}}, j \neq k} p_{j, n^{*}}\left\|\mathbf{H}_{k, n^{*}} \mathbf{W}_{j, n^{*}}\right\|_{F}^{2}+\sigma^{2}}
$$

The first term in the denominator of (10) is the interference from other UEs allocated on the same RB. Since this term is in the denominator, the function form is complicated, which makes it difficult to find the optimal value of $\mathbf{P}_{k, n^{*}}$ to maximize the energy efficiency. Notice that in the previous Sub-Section, we have determined the UEs on the same RB with lower cochannel interference. Besides, ZF beamforming is considered in this paper to further reduce the co-channel interference. Hence, the interference in (10) will be quite small and can be neglected, and then we can simplify (10) to the following formulation:

$$
\gamma_{k, n^{*}}=\frac{p_{k, n^{*}}\left\|\mathbf{H}_{k, n^{*}} \mathbf{W}_{k, n^{*}}\right\|_{F}^{2}}{\sigma^{2}}
$$

As for the objective function in (9), the difficulty is to obtain the best $\mathbf{P}_{k, n^{*}}$ which consists of a set of scalars. If the parameters $p_{1, n^{*}}, p_{2, n^{*}}, \ldots, p_{k, n^{*}}$ can be expressed as the functions of the same single variable, the maximum value of the objective function can be found much easier. To realize this idea, we introduce the classical water-filling power allocation algorithm. Set $g_{k, n^{*}}=\left\|\mathbf{W}_{k, n^{*}} \mathbf{H}_{k, n^{*}}\right\|_{F}^{2} / \sigma^{2}$, and the water-filling algorithm can be expressed as:

$$
p_{k, n^{*}}=\left(\alpha_{n^{*}}-\frac{1}{g_{k, n^{*}}}\right)^{+}
$$

where $(x)^{+}=\max (0, x)$, and $\alpha_{n^{*}}$ is the water-filling level, which ensures $\sum_{k \in \mathcal{A}_{n^{*}}} p_{k, n^{*}} \leq P_{n^{*}}$, where $P_{n^{*}}$ is the maximum transmit power limit on $\mathrm{RB} n^{*}$. Thus, the variables $p_{1, n^{*}}, p_{2, n^{*}}, \ldots, p_{k, n^{*}}$ can be unified to the function of $\alpha_{n^{*}}$. Then, we can rewrite the objective function as:

$$
\begin{aligned}
f\left(\alpha_{n^{*}}\right)= & \frac{\sum_{k \in \mathcal{A}_{n^{*}}} B \log _{2}\left(1+\gamma_{k, n^{*}}\right)}{\sum_{k \in \mathcal{A}_{n^{*}}} p_{k, n^{*}}+P_{n^{*}}^{(C)}} \\
= & \frac{B \sum_{k \in \mathcal{A}_{n^{*}}} \log _{2}\left(1+p_{k, n^{*}} g_{k, n^{*}}\right)}{\sum_{k \in \mathcal{A}_{n^{*}}} p_{k, n^{*}}+P_{n^{*}}^{(C)}} \\
= & \frac{B \sum_{k \in \mathcal{A}_{n^{*}}} \log _{2}\left(1+\left(\alpha_{n^{*}}-\frac{1}{g_{k, n^{*}}}\right)^{+} g_{k, n^{*}}\right)}{\sum_{k \in \mathcal{A}_{n^{*}}}\left(\alpha_{n^{*}}-\frac{1}{g_{k, n^{*}}}\right)^{+}+P_{n^{*}}^{(C)}}
\end{aligned}
$$

It was proved that the function in (13) is a quasi-concave function of $\alpha_{n^{*}}$ [15], and then we can find the maximum value of $f\left(\alpha_{n^{*}}\right)$ by differentiating $f\left(\alpha_{n^{*}}\right)$ with respect to $\alpha_{n^{*}}$ as shown in (14) shown at the bottom of the next page.

We solve $\frac{\partial f\left(\alpha_{n^{*}}\right)}{\partial \alpha_{n^{*}}}=0$ to obtain the solution of $\mathbf{P}_{k, n^{*}}$ The whole power allocation algorithm can be summarized as follow:

1) Obtain the results of $\mathcal{A}_{n^{*}}$ and $\rho_{k, n^{*}}$ from the user grouping method in Sub-section A.

2) On a specific $\operatorname{RB} n^{*}$, set the initial value of $\alpha_{\max }$ and $\alpha_{\min }$, the error threshold $\varepsilon$, and the maximum iteration number $D$. Set $\alpha_{n^{*}}=\left(\alpha_{\max }+\alpha_{\min }\right) / 2$. 
TABLE I

PARAMETERS OF SIMULATION

\begin{tabular}{|c|c|}
\hline System Parameters & Value \\
\hline Total bandwidth & $5 \mathrm{MHz}$ \\
\hline The number of RB $N$ & 25 \\
\hline $\begin{array}{c}\text { The number of transmit antennas on the } \\
\text { eNodeB } N_{t}\end{array}$ & 4 \\
\hline Channel model & $\mathrm{TU}$ \\
\hline Beamforming algorithm & $\mathrm{ZF}$ \\
\hline Maximum transmit power $P_{T}$ & $30 \mathrm{~W}$ \\
\hline
\end{tabular}

TABLE II

TARgET CHANNEL CAPACITY OF UES

\begin{tabular}{|c|c|}
\hline Target Rate & Number of UEs \\
\hline $128 \mathrm{kbps}$ & $10 \% \times K$ \\
\hline $256 \mathrm{kbps}$ & $20 \% \times K$ \\
\hline $512 \mathrm{kbps}$ & $30 \% \times K$ \\
\hline $1024 \mathrm{kbps}$ & $40 \% \times K$ \\
\hline
\end{tabular}

3) Calculate the derivate by (14). If $\frac{\partial f\left(\alpha_{n^{*}}\right)}{\partial \alpha_{n^{*}}} \leq 0$, set $\alpha_{\max }=$ $\alpha_{n^{*}}$, otherwise, set $\alpha_{\min }=\alpha_{n^{*}}$.

4) If $\alpha_{\max }-\alpha_{\min } \leq \varepsilon$, take the present value as $\alpha_{n^{*}}$ Otherwise, go back to (2) until the difference reaches the error threshold or the iteration number exceeds $D$.

5) Based on the final value of $\alpha_{n^{*}}$, calculate the value of $p_{k, n^{*}}$, and the energy efficiency and spectrum efficiency. Then, give a feedback to phase (4) in Sub-Section A to further select the UEs.

With the procedure of power allocation inside RBs, the energy efficiency can be improved. Combining these two Sub-Sections, the energy efficiency aware joint resource and power allocation algorithm is shown in algorithm 1.

The energy efficiency aimed power allocation is based on the results of the previous resource allocation. Hence, by this joint optimization algorithm, the energy efficiency of the whole system can be improved under the restriction that the data rate requirement of different UEs can be satisfied, and then, the spectrum efficiency and fairness of the system can also be guaranteed.

\section{Simulation AND ANALYSIS}

The proposed algorithm considers energy efficiency on the basis of spectrum efficiency, and in order to evaluate the performance of the proposed algorithm, the algorithms in [10] and
[22] and simplex power allocation scheme, in which a group of UEs are fixed on a given RB, are taken into comparison with the proposed algorithm. Ref. [10] proposed a resource allocation algorithm aiming to maximize the transmission data rate of UEs and spectrum efficiency in similar application scenarios as in our manuscript and its simulation shows that algorithm in [10] can achieve a very good performance of spectrum efficiency. Hence, we use [10] to verify that the proposed algorithm in this paper can provide better energy efficiency with very limited loss of spectrum efficiency. Meanwhile, Ref. [22] is our former work, which forced on system throughput with the consideration of fairness, and in this paper, we further extend the work considering the energy efficiency with the constraints of both user requirement and fairness. Therefore, we choose Ref. [22] as another benchmark for revealing the further improvement of our proposed algorithm.

Table I shows the main system parameters. In the simulations, the moving speed of UEs is $3 \mathrm{~km} / \mathrm{h}$, the traffic model is full buffer traffic, and the total circuit power consumption $P^{(C)}$ is set to be $12 \mathrm{~W}$. The pre-set target channel capacity $\left\{\lambda_{k}\right\}$ have been set to four different values according to the QoS requirements of different UEs and listed in Table II. The number of active UEs in the system $K$ is selected from the range of $[40,90]$.

Fig. 1 shows the energy efficiency performance of four algorithms with different UE number when the maximum transmit power $P_{T}$ is set to be $30 \mathrm{~W}$. From Fig. 1, it can be found that the energy efficiency of all these four algorithms increase with the increase of active UE number. This is because the possibility of selecting UEs with better channel conditions becomes bigger when the number of active UEs increases.

The performance of simplex resource allocation algorithm [22] and the algorithm in [10] are poor because they ignore the power optimization and almost all the power is used to obtain higher system throughput regardless the requirement of specific UE. Although the simplex power allocation algorithm focused on the optimization of power allocation, conventional Round Robin (RR) algorithm is used as the resource allocation scheme, which wastes system resource in some unnecessary UEs resulting in the decrease of system capacity, and hence its energy efficiency performance is also worse than the proposed algorithm.

Fig. 2 shows the energy efficiency of the system with different maximum transmit power constraint PT when the UE number

$$
\begin{aligned}
& \frac{\partial f\left(\alpha_{n^{*}}\right)}{\partial \alpha_{n^{*}}}=B \cdot \frac{d_{1} \cdot d_{3}-K_{n^{*}} \sum_{k=1}^{K_{n^{*}}} \log _{2}\left(1+g_{k, n^{*}}\left(\alpha_{n^{*}}-\frac{1}{g_{k, n^{*}}}\right)^{+}\right)}{d_{3}^{2}}, \alpha_{n^{*}}>\frac{1}{g_{k, n^{*}}} \\
& \frac{\partial^{2} f\left(\alpha_{n^{*}}\right)}{\partial \alpha_{n^{*}}{ }^{2}}=\frac{-B}{d_{3}{ }^{4}} \cdot\left\{d_{2} \cdot d_{3}{ }^{3}+2 \cdot K_{n^{*}} \cdot d_{3} \cdot\left[d_{1} \cdot d_{3}-K_{n^{*}} \sum_{k=1}^{K_{n^{*}}} \log _{2}\left(1+g_{k, n^{*}}\left(\alpha_{n^{*}}-\frac{1}{g_{k, n^{*}}}\right)^{+}\right)\right]\right\}, \alpha_{n^{*}}>\frac{1}{g_{k, n^{*}}},
\end{aligned}
$$

where,

$$
d_{1}=\sum_{k=1}^{K_{n^{*}}} \frac{1}{\ln 2} \cdot \frac{g_{k, n^{*}}}{1+g_{k, n^{*}}\left(\alpha_{n^{*}}-\frac{1}{g_{k, n^{*}}}\right)^{+}}, d_{2}=\sum_{k=1}^{K_{n^{*}}} \frac{1}{\ln 2} \cdot \frac{g_{k, n^{*}}^{2}}{\left[1+g_{k, n^{*}}\left(\alpha_{n^{*}}-\frac{1}{g_{k, n^{*}}}\right)^{+}\right]^{2}}, d_{3}=\sum_{k=1}^{K_{n^{*}}}\left(\alpha_{n^{*}}-\frac{1}{g_{k, n^{*}}}\right)^{+}+P_{n^{*}}^{(C)} .
$$




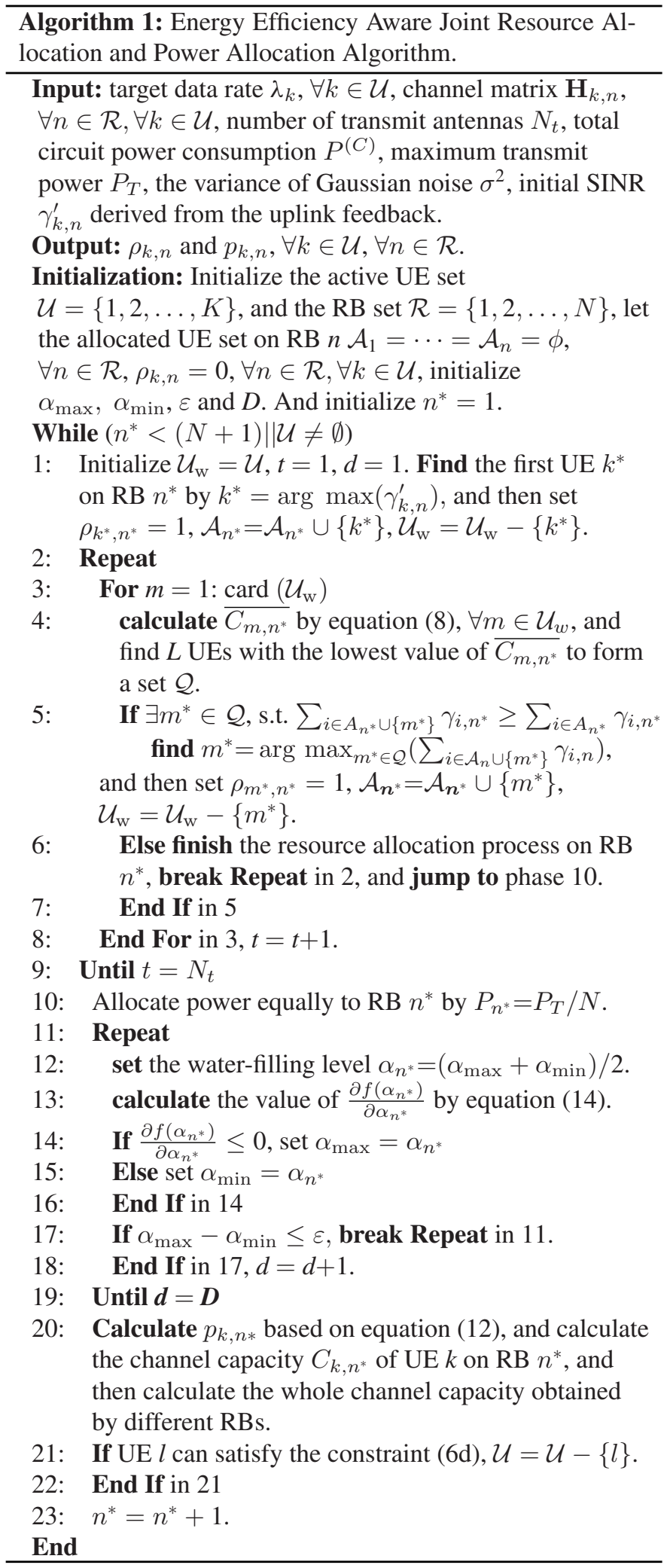

$\mathrm{K}=60$. Since the proposed algorithm can converge to an optimized power allocation parameter with the satisfaction of different UE requirement, the energy efficiency of the proposed algorithm is nearly unchanged with different maximum transmit power and remains the highest. However, energy efficiency

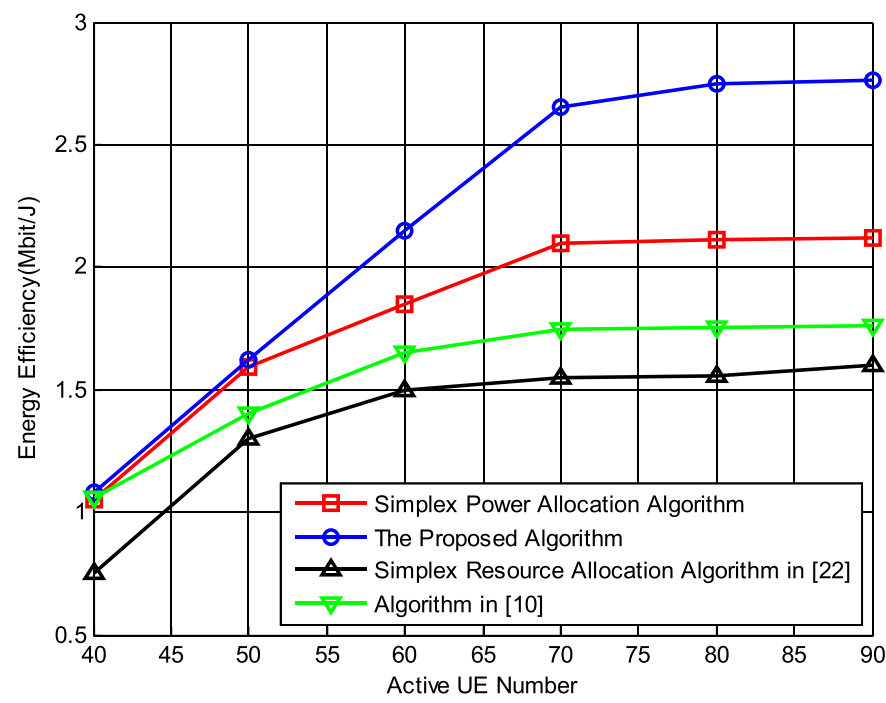

Fig. 1. Energy efficiency vs. active UE number when $P_{T}=30 \mathrm{~W}$.

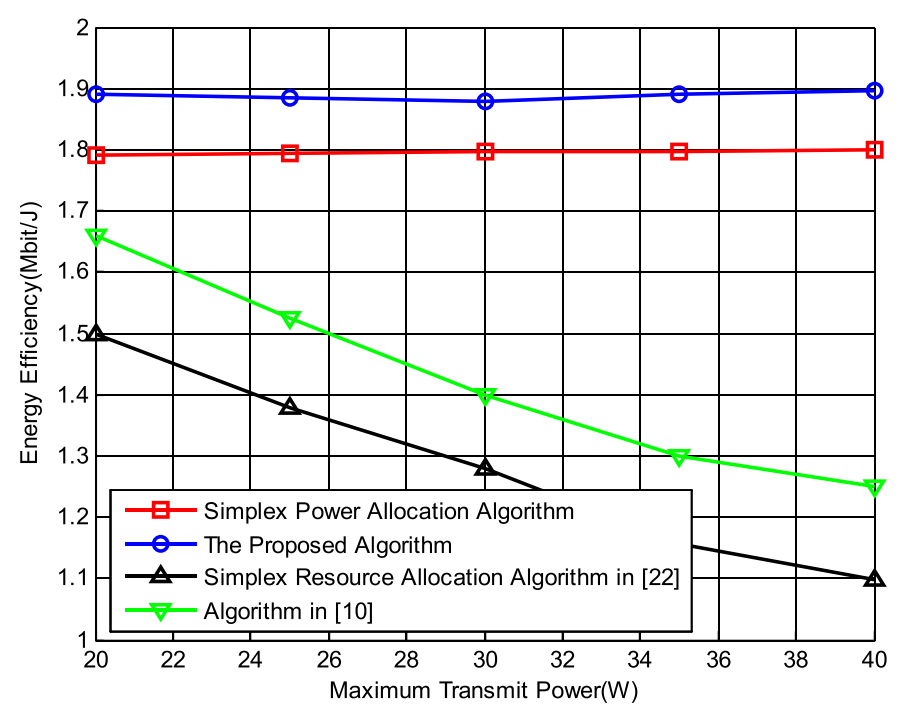

Fig. 2. Energy efficiency vs. maximum transmit power when $K=60$.

of the algorithm in [10] and the simplex resource allocation optimization algorithm in [22] drop with the increase of maximum transmit power due to the reason that they always use the maximum transmit power to transmit the signal. Since the RR resource allocation algorithm is used in the simplex power allocation algorithm, its energy efficiency may be further improved by using other efficient resource allocation algorithms.

Fig. 3 and Fig. 4 show the required transmit power with different active UE number and different maximum transmit power, respectively. In Fig. 3, power consumption of the proposed algorithm and the simplex power allocation algorithm decreases with the increase of active UE number. The reason is that as the number of UEs increases, more UEs with better channel conditions can be found, and then lower power consumption is required to satisfy the same transmission data rate requirement, e.g., when the number of active UEs reaches 90, the power consumption of the proposed algorithm and the simplex power allocation algorithm are only $4.3 \mathrm{~W}$ and $4.0 \mathrm{~W}$, respectively. The simplex power 


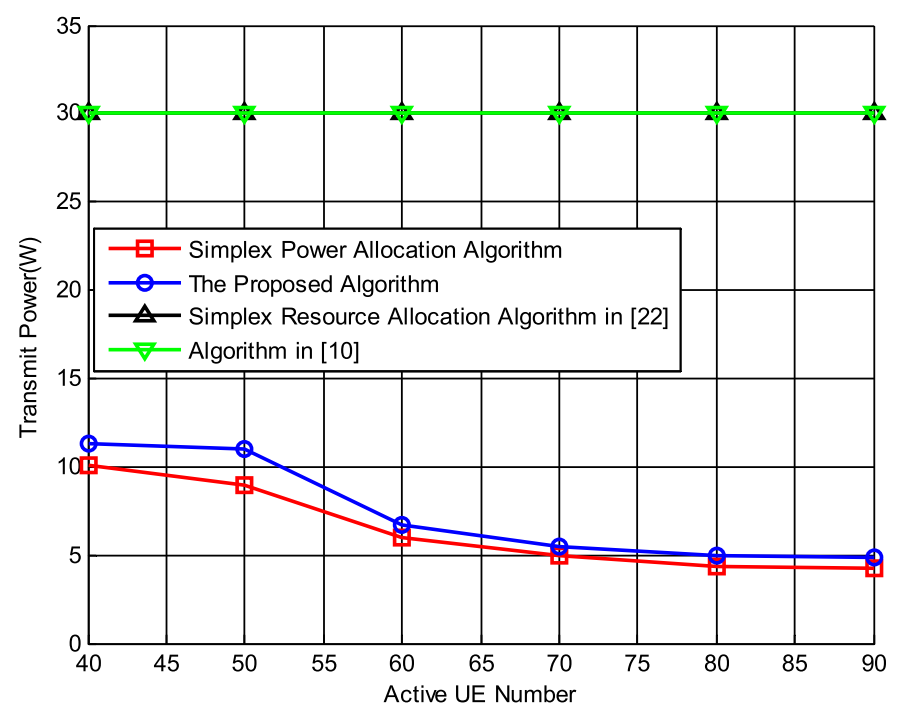

Fig. 3. Transmit power vs. active UE number when $P_{T}=30 \mathrm{~W}$.

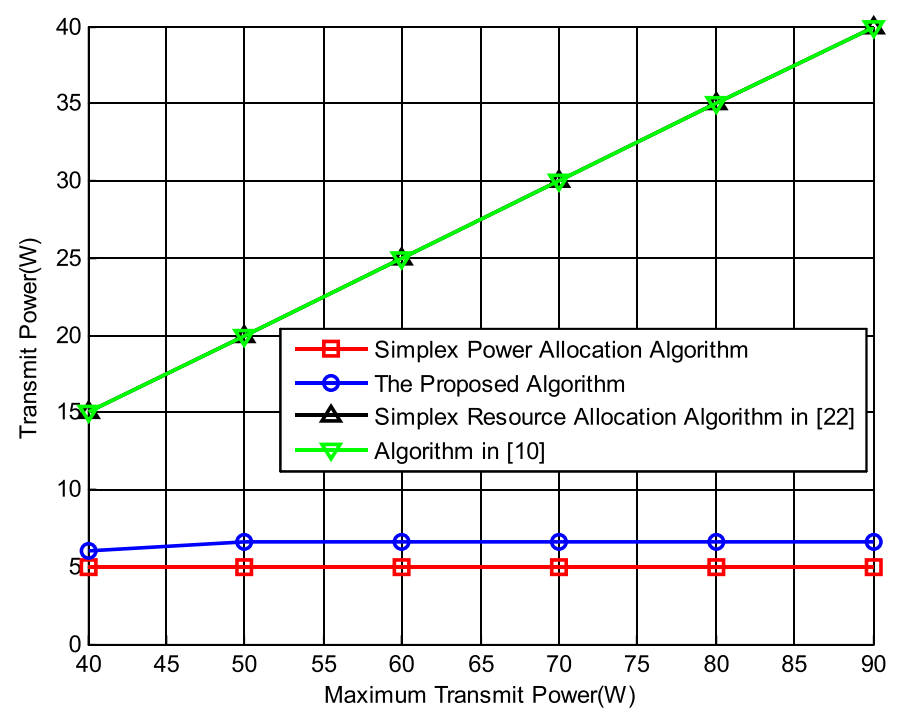

Fig. 4. Transmit power vs. maximum transmit power when $K=60$.

allocation algorithm uses less transmit power because of the lack of limitation on resource allocation which means that the QoS of less UE can be satisfied. In particular, with the increasing of UE number, the proposed algorithm can satisfy more UEs than the simplex power allocation algorithm. When the number of UE $K=90$, the number of UE whose data rate requirement is satisfied in the simplex power allocation algorithm is almost half of that in the proposed algorithm. Moreover, the simplex power allocation algorithm will have poor channel capacity because the channel conditions of different UEs are not considered in the power allocation process. Moreover, the algorithm in [10] and the simplex resource allocation algorithm result in a larger energy consumption because they always use the total transmit power. In Fig. 4, the transmit power of the proposed algorithm stops increasing after the best energy efficiency value, and thus the transmit power can be greatly reduced compared with the algorithms in [10] and [22].

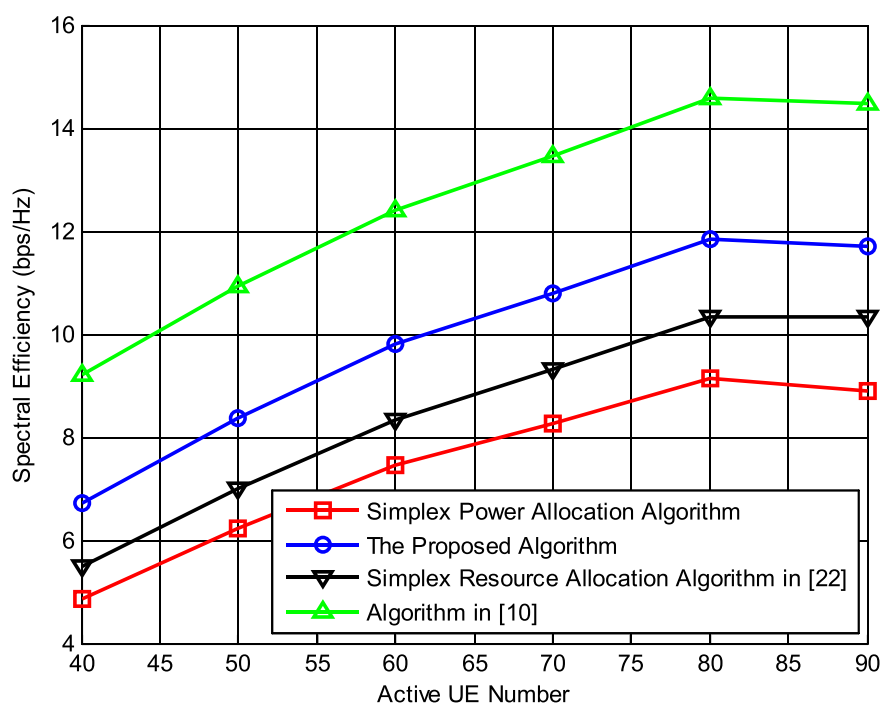

Fig. 5. Spectrum efficiency vs. active UE number when $P_{T}=30 \mathrm{~W}$.

The spectrum efficiency versus active UE number when $P_{T}=30 \mathrm{~W}$ with these four different algorithms are compared in Fig. 5. It can be found that the proposed algorithm shows higher spectrum efficiency than the simplex power allocation algorithm and the simplex resource allocation algorithm. Compared with the simplex power allocation algorithm, the proposed algorithm considers the resource allocation process which reduces the UE interference within each RB and enhances the UE SINR, and accordingly improves the spectrum efficiency of the UEs. When the number of UEs reaches 80 , the spectrum efficiency has been improved by $28 \%$ than the simplex power optimization algorithm. Compared with the simplex resource allocation algorithm, the proposed algorithm allocates power to the UE according to their channel status instead of equal power allocation, and hence the power consumption can be reduced and the total system throughput can thus be improved. Although the algorithm in [10] shows the best spectral efficiency among these four algorithms, from Fig. 1 and Fig. 2, we can find that its power efficiency is not very high, and total transmit power will be used to transmit signal.

Fig. 6 shows the system throughput comparison among these four algorithms in Rayleigh fading channel. The throughput here is not the Shannon channel capacity, but the actual transmission data rate of the system, which is calculated as follows:

$$
r_{k, n}=\left(N_{\text {sym }}-N_{\text {csym }}\right) \times Q m_{k, n} \times N_{\text {subcar }} \times R_{\text {code }}^{k, n},
$$

where, $r_{k, n}$ is the actual transmission rate of UE $k$ on RB $n$, $N_{\text {sym }}$ and $N_{\text {csym }}$ represents the total number of OFDM symbols and the number of OFDM symbols used to transmit the control signal within a TTI on each RB, respectively. $R_{\text {code }}^{k, n}$ and $Q m_{k, n}$ is the symbol rate and the number of bits per symbol modulation of UE $k$ on RB $n$, respectively, and both of them are decided by Modulation and Coding Scheme (MCS) of UE $k$ on $\mathrm{RB} n . N_{\text {subcar }}$ is the number of subcarriers on each RB, and in TD-LTE-A system, $N_{\text {subcar }}=12$. From Fig. 6 , we can find that the proposed algorithm shows better performance in terms of 


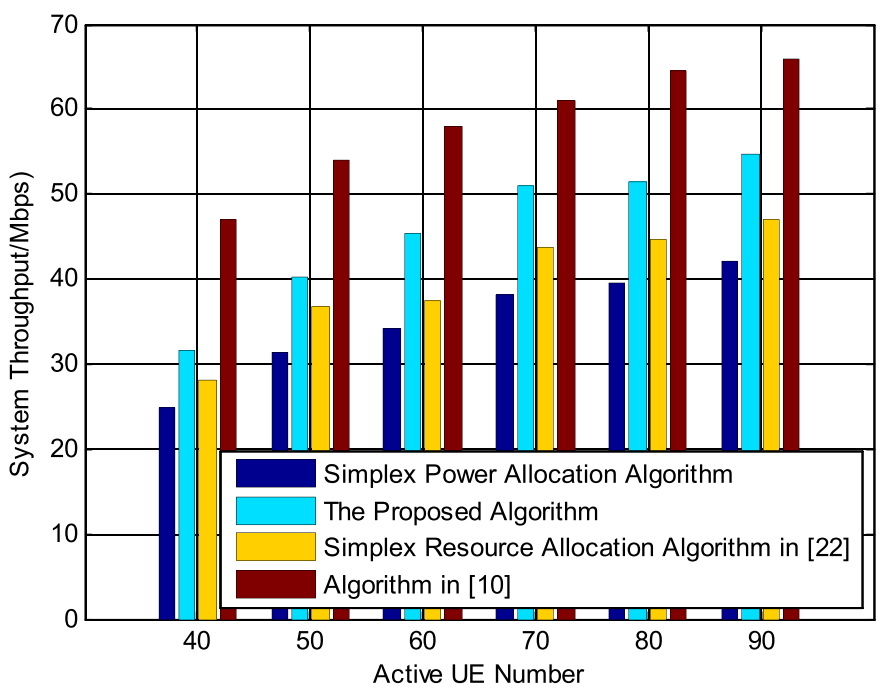

Fig. 6. Throughput vs. active UE number when $P_{T}=30 \mathrm{~W}$, Rayleigh Fading.

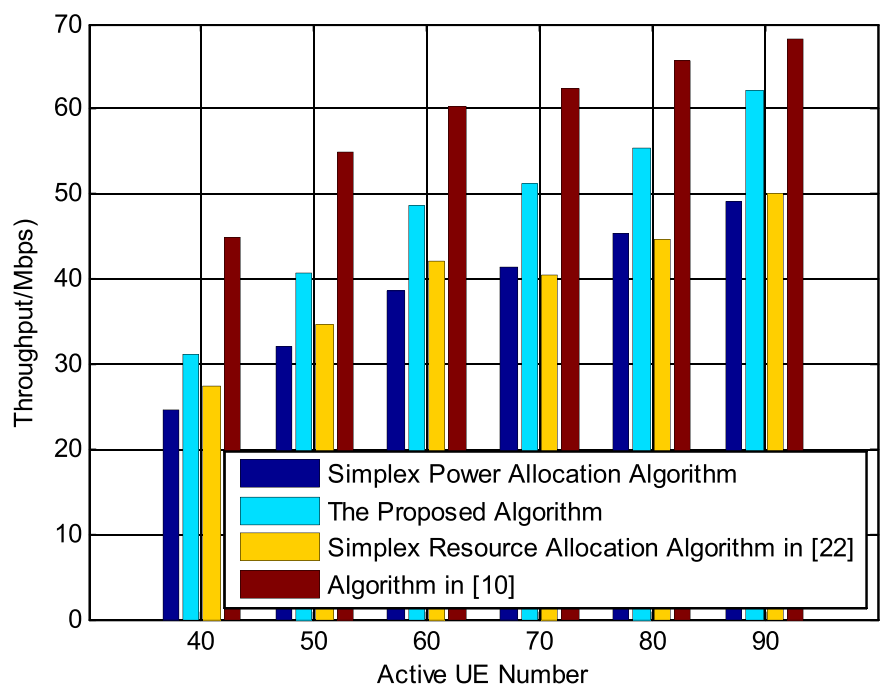

Fig. 7. Throughput vs. active UE number when $P_{T}=30 \mathrm{~W}$, Rician Fading.

throughput than the simplex power allocation algorithm and the simplex resource allocation algorithm. When the number of UEs is bigger than 80, more than $10 \mathrm{Mbps}$ improvement can be obtained by the proposed algorithm. The algorithm in [10] shows the best system throughput due to the same reason as explained in Fig 5. Meanwhile, we make the system throughput comparison in Rice fading channel as shown in Fig. 7 and the system throughput of our proposal can be further compared with that of the other three algorithms, especially the algorithm in [10]. As we could see from Fig. 7, when the channel changes from Rayleigh fading channel into Rician fading channel, the system throughput of algorithm in [10] is still the highest and the performance of our proposal also improves significantly, especially when the number of UE increases to 80. The algorithm in [10] shows the highest system throughput is due to the reason that it aims to maximize the transmission data rate by user selection and resource allocation, and the whole power can be allocated to the users regardless of their requirement. Meanwhile, we can

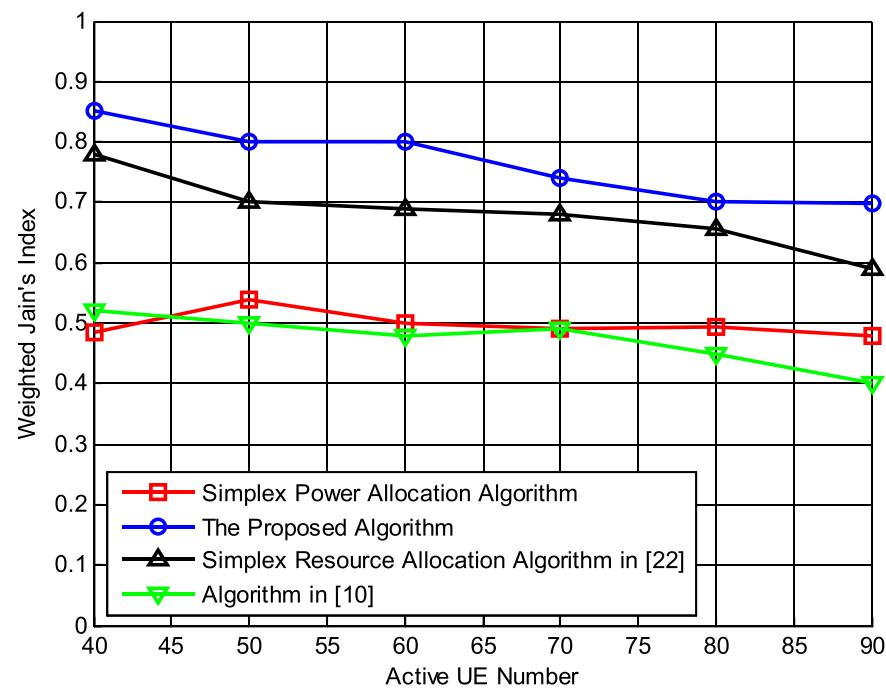

Fig. 8. Weighted Jain's index vs. active UE number.

also find that the performance gap between the algorithm in [10] and our proposal proposed algorithm becomes small, especially when the number of UE is bigger than 80, and this is due to the reason that with better channel conditions, more power will be allocated to UEs in our proposed algorithm for higher energy efficiency which leads to improved throughput, while the algorithm in [10] have exhausted all the power and cannot obtain significant improvement with better channel condition.

In Fig. 8, in order to evaluate the fairness performance, the weighted Jain's index, i.e., $F_{p}$ is shown with different active UE number, and $F_{p}$ is defined as follows:

$$
F_{P}=\frac{\left(\sum R_{k} / \lambda_{k}\right)^{2}}{K \sum\left(R_{k} / \lambda_{k}\right)^{2}},
$$

where, $R_{k}$ and $\lambda_{k}$ are the throughput and the target data rate of UE $k$, respectively. Different from traditional fairness index, $F_{p}$ represents the target data rate weighted fairness, which represents the system ability to satisfy different requirement of different UEs.

In Fig. 8, since the resource allocation process of both simplex power allocation algorithm and the algorithm in [10] does not take the requirement of different UEs into account, and UEs with poor channel conditions may unable to obtain any resource for transmission, and hence those two algorithms show very poor performance in terms of fairness. Although simplex resource allocation algorithm shows better fairness performance compared with the above two algorithms due to the reason that transmission data rate requirement of different UEs is considered in the process of resource allocation, the power allocation is not optimized so that the requirement of different UEs are not satisfied well. In comparison, the proposed algorithm shows the best fairness performance due to the reason that the transmission data rate requirement of different UEs is considered in the process of power allocation process, and more resources will be allocated to the UEs with higher transmission data rate requirement. When the active UE number is bigger than 50, only the proposed algorithm can provide the fairness performance higher than 0.7. 
To summarize, the proposed algorithm shows higher spectrum efficiency and energy efficiency, and it can also satisfy more UEs in the system with good fairness performance.

\section{CONCLUSION}

An energy efficiency aware joint resource and power allocation algorithm is proposed in this paper. It is applied in the MU-MIMO scenario, especially for the ZF or BD beamforming. The algorithm firstly shows a low-interference UE grouping algorithm, and then, it presents a resource allocation algorithm based on the results of the user grouping algorithm, which ensure the UEs with better channel quality can be properly allocated on corresponding RBs. In the resource allocation algorithm, the optimal water-filling level is calculated to improve the energy efficiency. Simulation results demonstrate that the proposed algorithm improves the energy efficiency and also shows a better performance in terms of spectrum efficiency. Besides, due to the comprehensive consideration of both channel quality and UE requirement in the proposed algorithm, both the number of satisfied UEs and fairness performance can be increased compared with other algorithms.

Since in 5G system, massive MIMO has been used, the extension of the proposed algorithm to massive MIMO scenario will be studied in our future work.

\section{REFERENCES}

[1] P. Gogoi, A. Sarma, R. Borah, and B. Saikia, "On the evolution of downlink physical layer in multi-antenna 3GPP LTE/LTE-A: A review," in Proc. Int. Symp. Adv. Comput. Commun., Silchar, 2015, pp. 290-296.

[2] H. M. E. Misilmani and A. M. El-Hajj, "Massive MIMO design for 5G networks: An overview on alternative antenna configurations and channel model challenges," in Proc. Int. Conf. High Perform. Comput. Simul., Genoa, 2017, pp. 288-294.

[3] F. Tang, Z. M. Fadlullah, N. Kato, F. Ono, and R. Miura, "AC-POCA Anti-coordination game based partially overlapping channels assignment in combined UAV and D2D based networks," IEEE Trans. Veh. Technol., vol. 67, no. 2, pp. 1672-1683, Feb. 2018

[4] N. Zhang, N. Lu, N. Cheng, J. W. Mark, and S. Shen, "Cooperative spectrum access towards secure information transfer for CRNs," IEEE J. Sel. Areas Commun., vol. 31, no. 11, pp. 2453-2464, Nov. 2013.

[5] F. Heliot, M. A. Imran, and R. Tafazolli, "On the energy efficiency-spectra efficiency trade-off over the MIMO Rayleigh fading channel," IEEE Trans. Commun., vol. 60, no. 5, pp. 1345-1356, May 2012.

[6] Q. Li et al., "MIMO techniques in WiMAX and LTE: A feature overview," IEEE Commun. Mag., vol. 48, no. 5, pp. 86-92, May 2010.

[7] T. M. Pham, R. J. Farrell, J. Dooley, E. Dutkiewicz, D. N. Nguyen, and L. N. Tran, "Efficient zero-forcing precoder design for weighted sumrate maximization with per-antenna power constraint," IEEE Trans. Veh. Technol., vol. 67, no. 4, pp. 3640-3645, Apr. 2018.

[8] V. F. Crâşmariu, M. O. Arvinte, A. A. Enescu, and S. Ciochină, "Performance analysis of the singular value decomposition with blockdiagonalization precoding in multi-user massive MIMO systems," in Proc. 12th IEEE Int. Symp. Electron. Telecommun., Timisoara, 2016, pp. 71-74.

[9] C. I, C. Rowell, S. Han, Z. Xu, G. Li, and Z. Pan, "Toward green and soft: A 5G perspective," IEEE Commun. Mag., vol. 52, no. 2, pp. 66-73, Feb. 2014.

[10] E. Castañeda, A. Silva, R. Samano-Robles, and A. Gameiro, "Lowcomplexity user selection for rate maximization in MIMO broadcast channels with downlink beamforming," Sci. World J., vol. 2014, 2014, Art. no. 865905 .

[11] W. Xu, R. Qiu, and J. Cheng, "Fair optimal resource allocation in cognitive radio networks with co-channel interference mitigation," IEEE Access, vol. 6, pp. 37418-37429, 2018

[12] T. Keenan and R. Villing, "Maximising spectral efficiency in LTE cells," in Proc. IET Irish Signals Syst. Conf., Maynooth, 2012, pp. 1-6.
[13] Y. Zhou, Z. M. Fadlullah, B. Mao, and N. Kato, “A deep learning based radio resource assignment technique for $5 \mathrm{G}$ ultra dense networks," IEEE Netw. Mag., vol. 32, no. 6, pp. 28-34, Nov./Dec. 2018.

[14] S. Zhang, N. Zhang, S. Zhou, J. Gong, Z. Niu, and X. Shen, "Energy-aware traffic offloading for green heterogeneous networks," IEEE J. Sel. Areas Commun., vol. 34, no. 5, pp. 1116-1129, May 2016.

[15] M. Patra, R. Thakur, C. Siva, and R. Murthy, "Improving delay and energy efficiency of vehicular networks using mobile femto access points," IEEE Trans. Veh. Technol., vol. 66, no. 2, pp. 1496-1505, Feb. 2017.

[16] R. S. Prabhu and B. Daneshrad, "An energy-efficient water-filling algorithm for OFDM systems," in Proc. IEEE Int. Conf. Commun., 2010, pp. $1-5$.

[17] C. Sun, J. Ge, J. Li, and B. Zhu, "Low complexity user scheduling algorithm for energy-efficient multiuser multiple-input multiple-output systems," IET Commun., vol. 8, no. 3, pp. 343-350, 2014.

[18] C. C. Hsu and J. M. Chang, "Spectrum-energy efficiency optimization for downlink LTE-A for heterogeneous networks," IEEE Trans. Mobile Comput., vol. 16, no. 5, pp. 1449-1461, May 2017.

[19] D. Wu, Q. Wu, Y. Xu, and Y. Liang, "QoE and energy aware resource allocation in small cell networks with power selection, load management, and channel allocation," IEEE Trans. Veh. Technol., vol. 66, no. 8, pp. 74617473, Aug. 2017.

[20] S. Li, Q. Ni, Y. Sun, G. Min, and S. Al-Rubaye, "Energy-efficient resource allocation for industrial cyber-physical IoT systems in $5 \mathrm{G}$ era," IEEE Trans. Ind. Inform., vol. 14, no. 6, pp. 2618-2628, Jun. 2018.

[21] G. Wu, C. Yang, S. Li, and G. Y. Li, "Recent advances in energy-efficient networks and their application in 5G systems," IEEE Wireless Commun., vol. 22, no. 2, pp. 145-151, Apr. 2015.

[22] X. Wu, Z. Ma, and Y. Wang, "Joint user grouping and resource allocation for multi-user dual layer beamforming in LTE-A," IEEE Commun. Lett., vol. 19 , no. 10 , pp. 1822-1825, Oct. 2015.

[23] V. D. Papoutsis, I. G. Fraimis, and S. A. Kotsopoulos, "User selection and resource allocation algorithm with fairness in MISO-OFDMA," IEEE Commun. Lett., vol. 14, no. 5, pp. 411-413, May 2011.

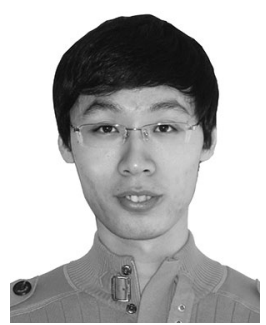

Xuanli Wu received the $\mathrm{Ph} . \mathrm{D}$. degree in communication and information system from the Harbin Institute of Technology, Harbin, China, in 2008. He is currently an Associate Professor with the School of Electronic and Information Engineering, Harbin Institute of Technology. His research interests include cooperative communications, ultra-dense networks, physical layer security for $5 \mathrm{G}$, and non-orthogonal multiple access technology. He is the Chair of IEEE Harbin VTS Chapter and was also a TPC member for many international conferences, including the IEEE ICC, GLOBECOM, VTC, INFOCOM, WCNC, and PIMRC.

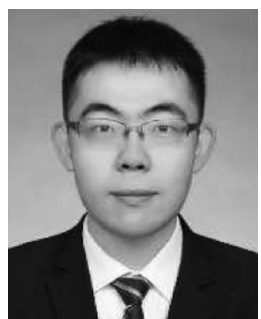

Zheming Ma received the bachelor's and master's degree from the School of Electronics and Information Engineering, Harbin Institute of Technology, Harbin, China, in 2014 and 2016, respectively. He is currently an Engineer with Ericsson (China) Communications Co., Ltd., Beijing, China, mainly working on the software development for $5 \mathrm{G}$ New Radio. His research interests include MIMO precoding, resource allocation in wireless network, and $5 \mathrm{G}$ air interface technologies. 


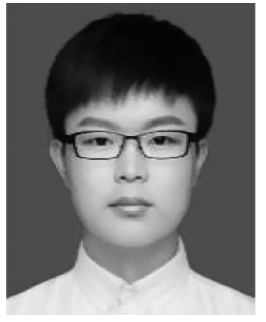

Xu Chen received the B.E. degree in communication engineering form the Harbin Institute of Technology, Harbin, China, in 2018, where he is currently working toward the master's degree. His research interests include wireless resource allocation and performance analysis of ultra-dense networks.

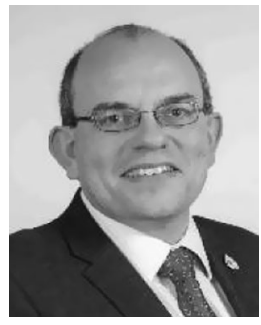

Fabrice Labeau is with McGill University, where he also holds the NSERC/Hydro-Québec Industrial Research Chair in Interactive Information Infrastructure for the Power Grid. He is the Director of Operations of STARaCom, an interuniversity research center grouping 50 professors and 500 researchers from ten universities in the province of Quebec, Canada. He has authored and coauthored more than 200 papers in refereed journals and conference proceedings. His research interests include applications of signal processing.

Mr. Labeau is the President of the Institute of Electrical and Electronics Engineers (IEEE) Sensors Council, Senior Past President of the IEEE Vehicular Technology Society, and the Past Chair of the Montreal IEEE Section. He was a recipient in 2015 and 2017 of the McGill University Equity and Community Building Award (team category), of the 2008 and 2016 Outstanding Service Award from the IEEE Vehicular Technology Society, and of the 2017 W.S. Read Outstanding Service Award form IEEE Canada.

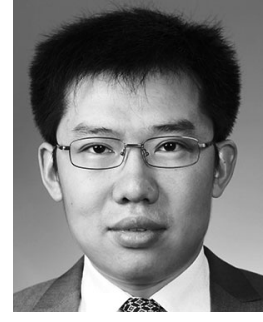

Shuai Han (S'11-M'12-SM'17) began the university studies in communication engineering in 2000 and received the M.E. and Ph.D. degrees in information and communication engineering from the Harbin Institute of Technology, Harbin, China, in 2007 and 2011, respectively. He completed his Postdoctoral work in electrical and computer engineering from the Memorial University of Newfoundland in Canada in 2012. He is currently an Associate Professor with the Department of Electronics and Communication Engineering, Harbin Institute of Technology. Over the academic career, he and his students have contributed in various fields in wireless networks and wireless positioning. His research interests include wireless sensor networks, wireless communications, the global navigation satellite system, and indoor location. 